\title{
Características Neuroanatómicas del Síndrome de Asperger
}

\author{
Neuroanatomical Characteristics of Asperger's Síndrome
}

Bélgica Vásquez ${ }^{1}$ \& Mariano del Sol $^{2.3}$

VÁSQUEZ, B. \& DEL SOL, M. Características neuroanatómicas del Síndrome de Asperger. Int. J. Morphol., 35(1):376-385, 2017.

RESUMEN: El síndrome de Asperger (SA) es un trastorno del neurodesarrollo que se caracteriza por presentar deterioros cualitativos de las interacciones sociales recíprocas y de los modos de comunicación, como también por la restricción del repertorio de intereses y de actividades que se aprecian estereotipadas y repetitivas. En la actualidad, el Manual Diagnóstico y Estadístico de Trastornos Mentales (DSM-V) decide eliminar esta subcategoría e incorporarla en una categoría general conocida como trastorno del espectro autista (TEA), lo que ha producido muchos debates y desacuerdos principalmente por distingirlo o no, con el autismo del alto funcionamiento (AAF). Un enfoque para resolver esta cuestión corresponde a los esfuerzos que se realizan por comprender la neuroanatomía estructural y funcional del TEA y del SA en particular, sin embargo, estas aproximaciones han dado lugar a resultados variables, debido a las diferencias en la edad, género, subcategorías y coefieciente intelectual de los sujetos de estudio, así como por criterios de inclusión y metodología de estudio. En base a lo anterior, el objetivo de esta revisión fue exponer el conocimiento actual de las características neuroanatómicas del SA considerando para esto aquellas investigaciones del TEA que individualizan este trastorno como entidad diagnóstica y lo diferencian del AAF.

PALABRAS CLAVE: Síndrome de Aspeger; Características neuroanatómicas.

\section{INTRODUCCIÓN}

El síndrome de Asperger (SA) fue descrito por primera vez por el médico psiquiatra y pediatra austríaco Hans Asperger, en 1944, bajo el nombre de "Psicopatía Autista" (Asperger, 1944). Sin embargo, la denominación de este trastorno fue acuñado por Lorna Wing en 1981 en un artículo publicado en la revista Psychological Medicine (Wing, 1981). Esta denominación se basó en el reconocimiento del trabajo previo realizado por Asperger y, porque la expresión "psicopatía autista" se prestaba a confusiones debido a asociación del término "psicopatía" con individuos que presentaban comportamientos psicopáticos y no al sentido técnico de una anomalía en la personalidad.

La entrada del SA en la historia de las psicopatologías ha estado marcada por controversias que lo ha llevado a una breve existencia como entidad diagnóstica (Barahona-Corrêa \& Filipe, 2016). Fue incluido por primera vez en la cuarta edición del Manual Diagnóstico y Estadístico de Trastornos Mentales (DSM-IV) (2000). Similar a los pacientes con autismo, los individuos con SA se caracterizaban por presentar deficiencias en la interacción social, habilidades de comunicación inadecuadas y restricción de intereses, pero también por una gran variedad de características clínicas sutiles, que para muchos, distinguen al SA del autismo (Wing, 1994; Witwer \& Lecavalier, 2008; Planche \& Lemonnier, 2012). En su individualidad, los paciente con SA debían presentar un desarrollo cognitivo y lingüístico normal antes de los 3 años y un funcionamiento adaptativo adecuado a la edad, en áreas distintas de la interacción social. Además, estos sujetos no debían cumplir con los criterios diagnósticos para el trastorno autista (TA), en cuyo caso, debe darse prioridad a este último diagnóstico, lo que implica un diagnóstico diferencial entre el SA y el autismo sin retraso cognitivo, también llamado autismo de alto funcionamiento (AAF) (Klin et al., 2005).

Sin embargo, las contradicciones en la definición del SA pronto se hicieron evidentes. El principal problema fue la prioridad dada al diagnóstico de autismo, ya que el requisito del desarrollo cognitivo y lingüístico normal no logró rescatar un diagnóstico del SA, por la sencilla razón de que el retraso cognitivo y lingüístico no es obligatorio para diagnosticar el TA (Mayes et al., 2001; Happé, 2011). Junto con lo anterior, la literatura señala que entre SA y AAF existe una gran similitud, ambas condiciones presentan diferentes gra-

\footnotetext{
${ }^{1}$ Facultad en Ciencias de la Salud, Universidad de Tarapacá, Chile.

${ }^{2}$ Centro de Excelencia en Estudios Morfológicos y Quirúrgicos, Universidad de La Frontera (CEMyQ), Temuco, Chile.

${ }^{3}$ Centro de Investigación en Estudios Biomédicos, Universidad Autónoma de Chile, Chile.
} 
dos de dificultad en la comunicación social, presencia de intereses inusualmente estrechos, resistencia al cambio y comportamientos altamente repetitivos (Ozonoff et al., 2000; Volkmar \& Partland, 2014).

En base a lo señalado anteriormente y a estudios clínicos (Macintosh \& Dissanayake, 2006) y biológicos (Lichtenstein et al., 2010), en el año 2013, el manual DSMV (última versión) eliminó la subcategoría de SA, clasificándola en una categoría general conocida como trastorno del espectro autista (TEA). Esta nueva clasificación destaca que los síntomas del SA y otros que también fueron incorporados como: el TA, el trastorno desintegrativo infantil y el trastorno generalizado del desarrollo no especificado, entre otros, representan un único continuo de alteraciones, de leves a graves, en los dos dominios de comunicación social y de conductas o intereses restringidos y repetidos, más que trastornos diferentes.

Por otra parte, la Clasificación Internacional de Enfermedades de la Organización Mundial de la Salud, en su última versión (CIE-10-ES) (1994), aún define al SA como una subcategoría del trastorno generalizado del desarrollo; caracterizado por deterioros cualitativos de las interacciones sociales recíprocas y de los modos de comunicación, como también por la restricción del repertorio de intereses y de actividades que se aprecian estereotipadas y repetitivas. En contraposición a lo señalado en el DSM-V, el CIE-10-ES señala que el SA difiere del TA y más específicamente del $\mathrm{AAF}$, fundamentalmente por que en el primero no hay retraso general, o retraso del desarrollo del lenguaje o del desarrollo intelectual, situación que ha sido apoyada por diversas investigaciones que dejan en evidencia patrones de anomalías neuroanatómicos diferentes entre el AAF y el SA (Kwon et al., 2004; Lotspeich et al., 2004; McAlonan et al., 2008, 2009). Adicionalmente, estudios recientes que utilizan la prueba de ojos, han demostrado diferencias entre SA y AAF en la capacidad de interpretar estados emocionales complejos en otros (Montgomery et al., 2016). Esta prueba presenta correlatos neuroanatómicos que incluyen la corteza prefrontal dorsal, la corteza frontal medial izquierda, el giro temporal superior y partes de la amígdala (Baron-Cohen et al., 1999; Richell et al., 2003; Holt et al., 2014). Las diferencias neuroanatómicas de algunas de estas estructuras entre ambas condiciones, también han sido reportadas en niños (McAlonan et al., 2008; Yu et al., 2011; Lai et al., 2015).

Las diferencias en los criterios diagnósticos, tanto en el DSM-V como en CIE-10-ES, son de considerable importancia a la hora de realizar un análisis de la literatura, en relación a las bases neuroanatómicas que expliquen los principales síntomas del SA. En la metodología de estas investi- gaciones se observa que las poblaciones estudiadas muestran diversidad en la clasificación diagnóstica, en la que se utilizan como sinónimos SA y AAF (Abell et al., 1999) o establecen que son condiciones distintas con superposición de síntomas conductuales, pero con un neurodesarrollo diferente (Lotspeich et al.; McAlonan et al., 2008, 2009; Yu et al., 2011). Además, en ocasiones los sujetos de estudio son heterogéneos, mostrando un amplio rango de severidad, pudiendo presentar asociadas otras posibles condiciones médicas, como déficit intelectual o deterioro en los lenguajes verbal y/o no verbal. Además, los grupos controles no siempre están emparejados adecuadamente por edad y nivel de coeficiente intelectual (Nickl-Jockschat et al., 2012), lo que hace difícil replicar los estudios, realizar comparaciones entre ellos e interpretar sus resultados.

En base a los antecedentes señalados, el objetivo de esta revisión fue exponer el conocimiento actual de las características neuroanatómicas del SA, considerando para esto aquellas investigaciones del TEA que individualizan este trastorno como entidad diagnóstica y lo diferencian del AAF.

\section{CARACTERÍSTICAS NEUROANATÓMICAS}

Cerebro. La primera observación del aumento del tamaño de la cabeza, que refleja la ampliación del cerebro, fue reportada en 1943 por Kanner, quien observó "cabezas grandes" en cinco de los once niños autistas que había examinado (Kanner, 1968). Posteriormente, un número creciente de estudios demostró que el aumento de la circunferencia cefálica fronto-occipital es uno de los hallazgos más persistentes en el autismo (Fombonne et al., 1999; Fidler et al., 2000; Aylward et al., 2002; Courchesne et al., 2003). Estas observaciones, junto con resultados de medidas métricas, peso del cerebro post-mortem y el volumen cerebral, a través de resonancia magnética, han determinado que la trayectoria de desarrollo del cerebro en los niños con TEA es muy diferente a los niños con desarrollo neurotípico (Redcay $\&$ Courchesne, 2005) y que estos efectos parecen depender de la edad (Courchesne et al., 2001; Courchesne et al., 2007), observándose un aumento pronunciado del perímetro de la cabeza y del volumen cerebral en periodos postnatal tempranos (aproximadamente entre 1 a 4 años de edad) (Courchesne $e t$ al., 2001; Hazlett et al., 2005;. Schumann et al., 2010) y ampliaciones del volumen cerebral cada vez menor en la última infancia y adolescencia (Courchesne et al., 2011; Duerden et al., 2012), siendo incluso comparables con los controles en edades más avanzadas (Aylward et al., 2002).

A la fecha, el crecimiento excesivo del cerebro en niños con TEA parece ser debido a aumentos tanto en la sustancia gris como blanca (Courchesne et al., 2001; Hazlett et al.). Sin embargo, los aumentos de sustancia gris se han 
evidenciado específicamente en los lóbulos frontales, temporales y parietales y han mostrado un patrón de crecimiento más típico en el lóbulo occipital (Palmen et al., 2005). Durante la adolescencia y edad adulta varias regiones del cerebro a nivel de giros, restringidos principalmente al lado izquierdo de la corteza temporal y parietal, son significativamente más delgadas (Wallace et al., 2010) y en las edades adulta media y tardía, los individuos con y sin TEA no muestran diferencias significativas en la sustancia gris (Koolschijn \& Geurts, 2016).

En el SA las anormalidades del volumen cerebral son específicas de cada región, varían con la edad y pueden permanecer dinámicas hasta la edad adulta. Al respecto, estudios que evalúan medidas morfométricas a través de voxel en imágenes de resonancia magnética de individuos con TEA y descripción clínica del SA, han mostrado diversos resultados. Kwon et al. observaron que varones con SA de 10 a 18 años de edad, presentaban disminución de la densidad de la sustancia gris en las regiones ventromedial de la corteza temporal y cuerpo del giro del cíngulo, en comparación con los controles emparejados por edad, sexo y coeficiente intelectual, señalando que lo observado apoya a las teorías que sugieren una implicación de estas áreas en la fisiopatología del autismo, en particular en la integración de estímulos visuales e información afectiva. Estudios adicionales en individuos adultos con SA señalaron que las alteraciones en el lóbulo temporal pueden estar asociadas también con una conectividad funcional anormal, especialmente en la corteza temporal medial, especializada en procesar estímulos faciales valentes afectivos (Welchew et al., 2005) y reconocimiento de caras (Pierce et al., 2004).

Por otra parte, Abell et al. observaron en individuos adultos con SA, disminución del volumen de sustancia gris en el surco paracingulado derecho, corteza occípito-temporal izquierda y surco frontal izquierdo y aumento del volumen de la corteza periamigdalina y giro temporal inferior derecho. Los autores señalaron que estas anormalidades estructurales forman parte de un circuito centrado en las amígdalas y que este sistema neuronal está fuertemente implicado en los aprendizajes emocional y social y más específicamente con la conciencia de sí mismos y de los otros.

McAlonan et al. (2002) en imágenes de resonancia magnética de cerebros de individuos adultos (18-49 años) con SA, observaron significativamente menor densidad de sustancia gris en las regiones fronto-estriada respecto de los controles. Los autores señalaron que estos resultados podrían estar asociados con anomalías de la supresión sensoriomotora, y en consecuencia, presentarían dificultades características relacionadas con la inhibición de pensamientos repetitivos, palabras y acciones.
Los estudios de resonancia magnética para evaluar volumen de sustancia blanca, también han mostrado resultados variados y regionalmente específicos. Por ejemplo, los niños (6 a 16 años) con SA presentaron significativamente más sustancia blanca en los circuitos subcorticales y lóbulo parietal del hemisferio izquierdo, en comparación con los controles. Sin embargo, tuvieron significativamente menos sustancia blanca en el lóbulo frontal derecho, especialmente alrededor del fascículo orbitofrontal derecho y rodilla del cuerpo calloso. Sin embargo, los volúmenes totales de tejido de sustancia blanca no fueron significativamente diferentes en comparación con los controles (McAlonan et al., 2009). Los autores señalaron que el mayor volumen de sustancia blanca del lóbulo parietal del hemisferio izquierdo podría deberse a una reorganización compensatoria de la sustancia blanca en niños con SA y que el menor volumen de sustancia blanca del lóbulo frontal del hemisferio derecho tiene diferencias relevantes con niños con AAF, ya que estos últimos presentan menor volumen de sustancia gris (McAlonan et al., 2008) y blanca (McAlonan et al., 2009) en el lóbulo frontal del hemisferio izquierdo lo que explicaría, al menos en parte, el por qué del retraso en la adquisición del lenguaje en este grupo y no en niños con SA.

McAlonan et al. (2002) observaron en adolescentes y adultos con SA, que el déficit de sustancia blanca estaba concentrado principalmente en el hemisferio izquierdo, en las regiones fronto-temporal y fronto-occipital, aparentemente dentro de tractos de fibras, tales como, los fascículos longitudinales inferiores y superiores y el fascículo occípitofrontal. Por el contrario, fue identificado una distribución bilateral de los excesos significativos de sustancia blanca en estas personas; esto se concentraba alrededor de los núcleos de la base y la cápsula externa. Según estos mismos autores, el hemisferio izquierdo normalmente se desarrolla más tarde que el derecho, lo que puede afectar particularmente a individuos con SA y, en consecuencia, provocar algunas de las anomalías del desarrollo del lenguaje.

Cerebelo. El cerebelo durante mucho tiempo ha despertado de gran interés ya que es una de las estructuras más consistentemente anormales en el TEA y puede conducir a una cascada de eventos, que directa o indirectamente, resultan en los síntomas conductuales y cognitivos asociados con este diagnóstico (McAlonan et al., 2005; Whitney et al., 2008; Fatemi et al., 2012; Rogers et al., 2013; Jumah et al., 2016). El cerebelo ha sido considerado como una región involucrada principalmente en el control motor y de coordinación (Glickstein et al., 2009). Sin embargo, estudios de lesiones cerebelares y de neuroimagen han demostrado que esta estructura también cumple una amplia gama de funciones neuropsicológicas del dominio cognitivo, tales como, la atención (Schweizer et al., 2007), la memoria de trabajo 
(Ferrucci et al., 2008), el aprendizaje (de Ribaupierre et al., 2008), el lenguaje (Lidzba et al., 2008) y las funciones ejecutivas (Leggio et al., 2008). Lesiones focalizadas del cerebelo han dado luces respecto a las regiones que parecen estar asociadas con estas habilidades. Daños del cerebelo lateral posterior se asociaron con deficiencias cognitivas, mientras que daños en la región cerebelar anterior fueron asociados con deficiencias motoras, manteniendo la cognición intacta (Exner et al., 2004). Se ha informado además, que lesiones del hemisferio derecho dan lugar a déficit en la memoria verbal y en el lenguaje, mientras que daños en el hemisferio izquierdo resultan en déficit del procesamiento visuoespacial (Townsend et al., 1999; Gottwald et al., 2004; Hokkanen et al., 2006).

Investigaciones que involucran al cerebelo en el TEA, han revelado resultados diversos, lo que demuestra el fenotipo heterogéneo de estos trastornos. Sin embargo, factores, tales como, la edad, el coeficiente intelectual, el sexo, los criterios de inclusión y la metodología de estudio, entre otros, deben ser considerados para una adecuada interpretación de los resultados. Algunos ejemplos de lo señalado son investigaciones de resonancia magnética que han informado mayor volumen en el hemisferio izquierdo (Boddaert et al., 2004), en el cerebelo anterior (Hyde et al., 2010) y volumen total (Hardan et al., 2001; McAlonan et al., 2005), en relación a los controles. Por el contrario, se ha reportado disminución del volumen cerebeloso de manera regionalmente específica (Courchesne et al., 1988; Courchesne et al., 1994; Kaufmann et al., 2003) o no se han encontrado diferencias en el volumen total (Piven et al., 1997).

Por otra parte, estudios han demostrado anomalías en los núcleos cerebelosos profundos como los núcleos fastigial, globoso y emboliforme (Williams et al., 1980), menor volumen del vermis cerebeloso en niños autistas, en comparación con niños de desarrollo típico (Webb et al., 2009 ) y desconexión cerebelosa, tanto interna como externa que podrían resultar no sólo en problemas de comunicación entre el cerebelo y sus objetivos eferentes, sino también, en compensaciones o adaptaciones de los circuitos neuronales afiliados (Belmonte et al., 2004; Allen et al., 2004). Junto con lo anterior, estudios post-mortem informaron números reducidos de neuronas purkinjenses (células de Purkinje) y procesos neuroinflamatorios activos dentro de la sustancia blanca del cerebelo (Bailey et al., 1998 ; Bauman \& Kemper, 2005b ; Vargas et al., 2005).

En el SA, las investigaciones también muestran controversias en el volumen cerebelar, aún cuando los individuos de estudio compartan el mismo sexo y edades similares. Abell et al. reportaron aumento bilateral en el volumen de sustancia gris del cerebelo, McAlonan et al. (2002) observaron disminución bilateral de sustancia gris en la misma estructura y, más tarde, Catani et al. (2008) no observaron diferencias significativas en el volumen cerebeloso, respecto del grupo control.

Por otra parte, se ha observado que en el TEA el cerebelo se encuentra particularmente implicado en el déficit de la conectividad y en la coordinación de las funciones cognitivas de largo alcance (Belmonte et al.). Esta estructura presenta múltiples conexiones aferentes y eferentes con regiones supratentoriales. El sistema aferente comprende las vías córtico-pontina y ponto-cerebelar, que implican la proyección de la información cortical a la núcleos pontinos y luego hacia el cerebelo. El sistema eferente consiste en el sistema cerebelo-talámico y los trayectos tálamo-corticales, que implican la proyección de información cerebelosa de los núcleos cerebelosos profundos al tálamo y luego a la corteza cerebral (Schmahmann, 2001). La vía cerebelo-tálamo-cortical tiene múltiples proyecciones corticales, con tramos de fibras dentro de las regiones motoras y no motoras de la corteza prefrontal (Middleton \& Strick, 2000, 2001; Kalmbach et al., 2009). Se han observado proyecciones aferentes de una serie de sitios de la corteza prefrontal hacia el cerebelo (Kelly \& Strick, 2003), demostrando reciprocidad entre ambas estructuras.

Bajo este contexto, investigaciones a través de tractografía de tensor de difusión de imágenes de resonancia magnética, han demostrado que existen proyecciones de retroalimentación del cerebelo alteradas en el SA (Catani $e t$ $a l$.). Los autores señalaron que la reducción de la anisotropía fraccional cerebelosa encontrada puede reflejar diferencias en la maduración y/o mielinización axonal en este síndrome, viéndose afectadas principalmente las fibras paralelas procedentes de las células granulares, las proyecciones de las neuronas purkinjenses (fibras intracerebelosas cortas) y el pedúnculo cerebeloso superior (principal salida del cerebelo). En base a lo observado, los autores también plantearon que es posible que la alteración de estos circuitos tengan un efecto distante en otras regiones de la sustancia blanca del cerebro.

En concordancia a lo señalado anteriormente, Carper y Courchesne (2000) observaron que niños con TA mostraban una correlación inversa entre el tamaño del lóbulo frontal y el cerebelo, de modo que el lóbulo frontal presentaba un exceso de tejido neural mientras que el cerebelo un déficit. Los autores plantearon que en este trastorno, las alteraciones cognitivas generalizadas y persistentes que lo caracterizan, pueden ser la consecuencia de una anomalía anatómica simultánea, no sólo en el lóbulo frontal, sino también, en el cerebelo. Por su parte, estudios neuroconductuales en individuos con SA y TA han demostrado asociaciones entre anormalidades anatómicas del cerebelo y disfunción 
sensoriomotora, cognitiva, lingüística, de aprendizaje y déficit social, las cuales son funciones atribuidas también al lóbulo frontal (Townsend et al.; Pierce \& Courchesne, 2001; Allen \& Courchesne , 2003; Gowen \& Miall, 2005; Strick et al., 2009; D`Mello \& Stoodley, 2015).

Amígdala. Los déficits en la función social representan una característica fundamental de los TEA y aunque la neuropatología aún no se ha establecido claramente, investigaciones han puesto de manifiesto alteraciones funcionales y morfológicas de la amígala en respuesta a este tipo de funciones (Baron-Cohen et al., 1999; 2000). Así, debido a que las expresiones faciales emocionales transmiten información no verbal que ayuda al andamio de las interacciones sociales, estudios del TEA han examinado la red de estructuras neuronales que incluyen a la amígdala en el procesamiento de la cara (Haxby et al., 2002), la atención conjunta (Charman et al., 1997; Sigman et al., 1986), el reconocimiento de las emociones (Adolphs, 2002; Adolphs \& Tranel, 2003; Welchew et al.) y la mejora de la memoria para los eventos emocionales significativos (Canli et al., 2000).

Volúmenes de la amígdala anormales se han observado en varios estudios de resonancia magnética estructural en niños, adolescentes y adultos con TEA. Schumann et al. (2004) sugirieron por primera vez, que las inconsistencias entre los estudios son el resultado de los efectos dependientes de la edad. Los autores observaron que la amígdala de individuos autistas con edades entre 12 a 18 años, es aproximadamente del mismo tamaño que sus controles. Sin embargo, la amígdala de individuos autistas con edades entre 7 a 12 años, alcanza el tamaño adulto antes de la adolescencia, mientras que los niños de desarrollo típico presentan un crecimiento progresivo de la amígdala hasta la adolescencia. En consonancia con esta investigación Mosconi et al. (2009) observaron la ampliación bilateral de la amígdala, en una amplia gama de muestras de niños con TA de 2 años de edad, que persistió hasta los 4 años. Por su parte, Sparks et al. (2002) también observaron mayores volúmenes de la amígdala, en niños con TA de 3 a 4 años de edad. Por el contrario, estudios en adultos con autismo han puesto de relieve la reducción de volúmenes en la amígdala en comparación a los individuos con desarrollo normal (Aylward et al., 1999; Pierce et al., 2001).

Junto con lo anterior, algunos estudios han sugerido que la magnitud de la ampliación de la amígdala también se relaciona con el diagnóstico clínico y con la gravedad de sus deficiencias sociales y de comunicación (Schumann $e t$ al., 2009). Sparks et al. encontraron que cuando el grupo del espectro autista se diferenció en TA y en trastorno generalizado del desarrollo no especificado, los niños con TA tenían una amígdala aún mayor que aquellos con trastorno generalizado del desarrollo no especificado. En niños con diagnóstico de TA, la amígdala derecha era un $14 \%$ más grande y la izquierda un $12 \%$ mayor, en comparación con los niños con diagnóstico de trastorno generalizado del desarrollo no especificado. Otras investigaciones en base ha imágenes de resonancia magnética estructural, han mostrado que niños pequeños con TA tienen en promedio un $16 \%$ más grande la amígdala que los controles, mientras que los niños con SA tiene sólo un $9 \%$ más grande la amígdala que los controles (Schumann et al., 2004).

Estudios que intentan identificar diferencias neuroanatómicas entre el TA y el SA, han identificado que no existen diferencias significativas en la densidad de sustancia gris de la amígdala de adolescentes (10-18 años) con AAF y SA. Además, estos individuos tampoco mostraron diferencias significativas con el grupo control (Kwon et al.). Estos resultados se correlacionaron positivamente con lo observado por Schumann et al. (2004), quienes no encontraron diferencias significativas en el volumen de la amígdala entre adolescentes (12,75-18,5 años) con TA, SA y controles. Recientemente, Murphy et al. (2012) a través de imágenes de resonancia magnética estructural, realizaron comparaciones del volumen de la amígdala de 32 individuos con SA (12-47 años) y 32 controles sanos, quienes no difirieron significativamente en la edad o el coeficiente intelectual. Los hallazgos encontrados apoyaron informes previos de la ampliación de amígdala en niños pequeños con TEA (Sparks et al.; Schumann et al., 2004, 2009; Mosconi et al.); sin embargo, encontraron que en varones adolescentes y adultos con SA, la amígdala sigue siendo significativamente mayor, en comparación con los controles de la misma edad. Además, observaron que el desarrollo de la amígdala parecía ser diferente entre los grupos. La amígdala de los controles continuaba aumentando con la edad, mientras que la amígdala de los individuos con SA no lo hacía. En base a lo anterior, los autores sugirieron que tanto el volumen como el envejecimiento de la amígdala son significativamente diferentes en individuos con SA, de los controles y, por tanto, se necesitan estudios longitudinales futuros para ayudar a aclarar el desarrollo y el envejecimiento de la amígdala durante toda la vida útil, y además, para determinar si esto difiere a través de las subcategorías de diagnóstico del TEA.

Las causas de los patrones de crecimientos no lineales de la amígdala en el TEA y, por tanto en el SA, son desconocidas. Probablemente incluya una compleja interacción entre las diferencias genéticas en el desarrollo cerebral (Dalton et al., 2007; Dorris et al., 2004) y las interacciones anormales entre el individuo afectado y su entorno (Ghaziuddin, 2010; Montgomery et al.).

Hipocampo. El hipocampo, además de su rol en el procesamiento emocional y la memoria (Jin \& Maren, 2015), 
también participa en el aprendizaje (Mattfeld \& Stark, 2015) y junto con la amígdala forman una relación recíproca durante las tareas sociales y la memoria (Adolphs; Richardson et al., 2004; Richter-Levin, 2004).

Aunque los primeros relatos clínicos de personas con TA mencionaban sobre su buena memoria, estudios sistemáticos describen una situación más compleja del ahorro y daño en el proceso de memoria. Investigaciones en individuos adultos con SA demostraron déficit en la tarea de recuerdo libre inesperado (Bowler et al., 1997). Estudios posteriores (Bowler et al., 2000a; Bowler et al., 2000b) reportaron que en presencia de un reconocimiento global, existía un pequeño, pero significativo, déficits en el tipo de experiencia recordada y auto-participación en el recuerdo del episodio, necesario durante el proceso del sistema de memoria episódica (Tulving ,1985). En adolescentes y adultos con SA se observó dificultades en la memoria generalizada, la cual se manifestaba en cualquier tarea que requiera recordar algún elemento sin instrucciones específicas o sin un apoyo del entorno durante la prueba (Bowler et al., 2004).

En consideración a lo señalado, se ha sugerido que el TEA, incluyendo el SA, presenta anomalías en el desarrollo y la función del hipocampo (Dager et al., 2007; Salmond et al., 2005). Hay relativamente pocos estudios de neuroimagen in vivo del desarrollo normal del hipocampo y la mayoría de los estudios han sido transversales (Giedd $e t$ al., 1996). Sin embargo, los que están disponibles sugieren que el volumen del hipocampo en el desarrollo humano típico cambia con la edad. Por ejemplo, el volumen del hipocampo en el sexo masculino se mantiene durante la adolescencia (Giedd et al.; Schumann et al., 2004), aumenta en el adulto joven (19-21 años), (Suzuki et al., 2005), pero disminuye en la edad avanzada (Liu et al., 2003; Pruessner et al., 2000; Raz et al., 2005). En el TEA, si bien la edad es un factor importante, el subtipo, el coeficiente intelectual y los criterios de exclusión (por ejemplo, la presencia o ausencia de epilepsia) de las personas estudiadas, son factores que también se deben considerar en la interpretación de los resultados. En niños y adolescentes con TA se ha informado un hipocampo agrandado (Sparks et al.; Schumann et al., 2004; Groen et al., 2010) y en adultos se ha reportado sin diferencias (Piven et al., 1997) o disminución significativa (Aylward et al., 1999) respecto del grupo control.

Datos post-mortem publicados de individuos con TA (de 9 años de edad hasta adultos) han documentado anomalías anatómicas en el sistema límbico y en el hipocampo (Bauman \& Kemper, 2005a). Cuando tales alteraciones estaban presentes, hubo un aumento de la densidad de las células piramidales (número de neuronas por volumen) y reducción del tamaño de las células neuronales del hipocampo.
Análisis con tinción de Golgi de las neuronas piramidales de CA1 y CA4 demostraron una disminución de la complejidad y extensión de la arborización dendrítica en estas células (Raymond et al., 1989).

Estudios realizados en niños y adolescentes con SA no identificaron correlaciones significativas entre la edad y el volumen del hipocampo y no encontraron diferencias significativas con los controles (Schumann et al., 2004). Investigaciones posteriores señalaron resultados similares en adolescentes y adultos con este síndrome (O'Brien et al., 2010; Murphy et al., 2012). Sin embargo, Toal et al. (2010) y Yu et al. observaron reducción de la sustancia gris en hipocampos de individuos adultos con SA en comparación con los controles.

\section{Consideraciones futuras y conclusiones}

En la actualidad, los estudios de imágenes estructurales y funcionales están convergiendo en la hipótesis de que el TEA se asocia con alteraciones en el volumen y densidades de sustancia gris y blanca de las estructuras encefálicas, así como la presencia de conectividad atípica, lo que conlleva probablemente a la producción de un sistema que es ineficaz para el procesamiento de información más compleja. Aunque este tipo de estudios nos ha entregado información acerca de la neurobiología del TEA y, del SA en particular, todavía tenemos que comprender este fenómeno en el contexto de la neurobiología del desarrollo, ya que la mayoría de las investigaciones realizadas han evaluado el efecto de la edad comparando grupos de personas por encima y por debajo de determinadas edades; concentrándose los resultados principalmente en niños, adolescentes y adultos jóvenes con TEA, con pocos antecedentes de individuos de la primera infancia y adultos mayores. Por tanto, estudios in vivo de tipo longitudinal con tamaños de muestras más grandes se tornan relevantes al momento de comprender la neuroanatomía asociada a características clínicas y síntomas cognitivos de este espectro. Además, el uso de técnicas automatizadas modernas, junto con la caracterización cuidadosa de los sujetos, probablemente disminuyan los resultados inconsistentes en la literatura.

En relación a las investigaciones en sujetos con SA las brechas del conocimiento son aún mayores. Los estudios estructurales de resonancia magnética que comparan el SA y el AAF han producido resultados contradictorios. Existe evidencia creciente que sugiere que las dos condiciones se pueden distinguir en el nivel neuroanatómico (Barahona-Corrêa \& Filipe). Yu et al. realizaron un metanálisis de la neuroanatomía de las personas con SA comparándola con las personas con AAF, utilizando la morfometría basada en voxel, encontrando diferencias 
significativas en el volumen de sustancia gris entre los grupos con patrones de distribución distintos. Aunque una revisión sistemática de los datos estructurales de resonancia magnética sugiere una distinción menos clara (PinaCamacho et al., 2013), los autores también concluyeron que, basándose en la evidencia disponible, puede ser demasiado pronto para eliminar las subcategorías del TEA en el DSMV. Por el contrario, en la literatura existen estudios que solo observaron diferencias cuantitativas, con valores del SA intermedios entre el AAF y los sujetos típicos y anormalidades estructurales comunes entre ambos trastornos que apoyan la propuesta de una única categoría del TEA en el DSMV (Lotspeich et al.; Haznedar et al., 2006).

Para dilucidar estas controversias, las investigaciones futuras del TEA deberían dirigirse hacia el suministro de información sensible y específica de biomarcadores que, en combinación con las pruebas de comportamiento, podría conducir más fácilmente a un diagnóstico preciso y temprano del trastorno (Pina-Camacho et al.). En general, estudios multi-céntricos y multi-modales basados en grandes grupos de pacientes es una de las estrategias clave para aumentar la probabilidad de descubrir nuevos biomarcadores efectivos que caractericen de manera integral las características clínicas y síntomas cognitivos del Síndrome de Asperger (Ruggeri et al., 2014; Li et al., 2017).

VÁSQUEZ, B. \& DEL SOL, M. Neuroanatomical Characteristics of Asperger’s Sindrome. Int. J. Morphol., 35(1):376-385, 2017.

RESUMEN: The asperger syndrome (AS) is a neurodevelopmental disorder that is characterized by qualitative deterioration of reciprocal social interactions and communication methods, as well as by the restriction of the repertoire of interests and activities that are perceived as stereotyped and repetitive. Actually, the Diagnostic and Statistical Manual of Mental Disorders (DSM-V) decides to eliminate this subcategory and to incorporate it into a general category known as autism spectrum disorder (ASD), which has produced many debates and disagreements mainly to distinguish it or not with High Functioning Autism (HFA). One approach to address this question is the effort to understand the structural and functional neuroanatomy of ASD and AS in particular; however, this approach has led to variable outcomes, often due to differences in age, gender, subcategories and IQ of study subjects, as well as by inclusion criteria and study methodology. Based on the above-mentioned, the aim of this review was to present the current knowledge of the neuroanatomical characteristics of AS considering for this the investigations of the ASD that individualize this disorder as a diagnostic entity and differentiate it from HFA.

KEY WORDS: Asperger syndrome; Neuroanatomical characteristics

\section{REFERENCIAS BIBLIOGRÁFICAS}

Adolphs, R. Neural systems for recognizing emotion. Curr. Opin. Neurobiol., 12(2):169-77, 2002.

Adolphs, R. \& Tranel, D. Amygdala damage impairs emotion recognition from scenes only when they contain facial expressions. Neuropsychologia, 41(10):1281-9, 2003.

Abell, F.; Krams, M.; Ashburner, J.; Passingham, R.; Friston, K.; Frackowiak, R.; Happé, F.; Frith, C. \& Frith, U. The neuroanatomy of autism: a voxel-based whole brain analysis of structural scans. Neuroreport, 10(8):1647-51, 1999.

Allen, G. \& Courchesne, E. Differential effects of developmenta lcerebellar abnormality on cognitive and motor functions in the cerebellum: an fMRI study of autism. Am. J. Psychiatry, 160(2):262-73, 2003.

Allen, G.; Müller, R. A. \& Courchesne, E. Cerebellar function in autism: functional magnetic resonance image activation during a simple motor task. Biol. Psychiatry, 56(4):269 -78, 2004.

American Psychiatric Association (APA). Diagnostic and statistical manual of mental disorders. $4^{\text {th }}$ ed. Washington, DC: APA, 2000.

American Psychiatric Association (APA). Diagnostic and statistical manual of mental disorders. $5^{\text {th }}$ ed., Arlington, VA: APA, 2013.

Asperger, H. Die "Autistischen Psychopathen" im Kindesalter. Archiv für Psychiatrie und Nervenkrankheiten, 117:76-136, 1944.

Aylward, E. H.; Minshew, N. J.; Goldstein, G.; Honeycutt, N. A.; Augustine, A. M.; Yates, K. O.; Barta, P. E. \& Pearlson, G. D. MRI volumes of amygdala and hippocampus in non-mentally retarded autistic adolescents and adults. Neurology, 53(9):2145-50, 1999.

Aylward, E. H.; Minshew, N. J.; Field, K.; Sparks, B. F. \& Singh, N. Effects of age on brain volume and head circumference in autism. Neurology, 59(2): 175-83, 2002

Bailey, A.; Luthert, P.; Dean, A.; Harding, B.; Janota, I.; Montgomery, M.; Rutter, M. \& Lantos, P. A clinicopathological study of autism. Brain, 121( Pt 5):889-905, 1998.

Barahona-Corrêa, J. B. \& Filipe, C. N. A Concise history of asperger syndrome: the short reign of a troublesome diagnosis. Front. Psychol., 6:2024, 2016.

Baron-Cohen, S.; Ring, H. A.; Wheelwright, S.; Bullmore, E. T.; Brammer, M. J.; Simmons, A. \& Williams, S. C. Social intelligence in the normal and autistic brain: an fMRI study. Eur. J. Neurosci., 11(16):1891-8, 1999.

Baron-Cohen, S.; Ring, H. A.; Bullmore, E. T.; Wheelwright, S.; Ashwin, C. \& Williams, S. C. The amygdala theory of autism. Neurosci. Biobehav. Rev., 24(3):355-64, 2000.

Bauman, M.; Kemper, T. L. The Neurobiology of Autism. $2^{\text {th }}$ ed. Baltimore: The Johns Hopkins University Press, Project MUSE, 2005a.

Bauman, M. L. \& Kemper, T. L. Neuroanatomic observations of the brain in autism: a review and future directions. Int. J. Dev. Neurosci., 23(23):183-7, 2005b.

Belmonte, M. K.; Allen, G.; Beckel-Mitchener, A.; Boulanger, L. M.; Carper, R. A. \& Webb, S. J. Autism and abnormal development of brain connectivity. J. Neurosci., 24(42):9228-31, 2004.

Boddaert, N.; Chabane, N.; Gervais, H.; Good, C. D; Bourgeois, M.; Plumet, M. H.; Barthelemy, C.; Mouren, M. C.; Artiges, E.; Samson, Y.; Brunelle, F.; Frackowiak, R. S. \& Zilbovicius, M. Superior temporal sulcus anatomical abnormalities in childhood autism: a voxel-based morphometry MRI study. NeuroImage, 23(1):364-9, 2004.

Bowler, D. M.; Matthews, N. J. \& Gardiner, J. M. Asperger's syndrome and memory: Similarity to autism but not amnesia. Neuropsychologia, 35(1):65-70, 1997.

Bowler, D. M.; Gardiner, J. M. \& Grice, S. Episodic memory and remembering in adults with Asperger's syndrome. J. Autism Dev. Disord., 30(4):305-16, 2000a.

Bowler, D. M.; Gardiner, J. M.; Grice, S. \& Saavalainen, P. Memory illusions: false recall and recognition in adults with Asperger's syndrome. J. Abnorm. Psychol., 109(4):663-72, 2000b. 
Bowler, D. M.; Gardiner, J. M. \& Berthollier, N. Source memory in adolescents and adults with Asperger's syndrome. J. Autism Dev. Disord., 34(5):533-42, 2004.

Canli, T.; Zhao, Z.; Brewer, J.; Gabrieli, J. D. \& Cahill, L. Event-related activation in the human amygdala associates with later memory for individual emotional experience. J. Neurosci., 20(19):RC99(1-5), 2000.

Charman, T.; Swettenham, J.; Baron-Cohen, S.; Cox, A.; Baird, G. \& Drew, A. Infants with autism: an investigation of empathy, pretend play, joint attention, and imitation. Dev. Psychol., 33(5):781-9, 1997.

Carper, R. A. \& Courchesne, E. Inverse correlation between frontal lobe and cerebellum sizes in children with autism. Brain, 123( Pt 4):836-44, 2000.

Catani, M.; Jones, D. K.; Daly, E.; Embiricos, N.; Deeley, Q.; Pugliese, L.; Curran, S.; Robertson, D.; Murphy, D. G. Altered cerebellar feedback projections in Asperger syndrome. Neuroimage 41(4):1148-91, 2008.

Courchesne, E.; Yeung-Courchesne, R.; Press, G. A.; Hesselink, J. R. \& Jernigan, T. L. Hypoplasia of cerebellar vermal lobules VI and VII in autism. N. Engl. J. Med., 318(21):1349-54, 1988.

Courchesne, E.; Saitoh, O.; Townsend, J. P.; Yeung-Courchesne, R.; Press, G. A.; Lincoln, A. J.; Haas, R. H. \& Schriebman, L. Cerebellar hypoplasia and hyperplasia in infantile autism. Lancet, 343(8888):634, 1994.

Courchesne, E.; Karns, C. M.; Davis, H. R.; Ziccardi, R.; Carper, R. A.; Tigue, Z. D.; Chisum, H. J.; Moses, P.; Pierce, K.; Lord, C.; Lincoln, A. J.; Pizzo, S.; Schreibman, L.; Haas, R. H.; Akshoomoff, N. A. \& Courchesne, R. Y. Unusual brain growth patterns in early life in patients with autistic disorder: an MRI study. Neurology, 57(2):245-54, 2001.

Courchesne, E.; Carper, R. \& Akshoomoff, N. Evidence of brain overgrowth in the first year of life in autism. JAMA, 290(3):337-44, 2003.

Courchesne, E.; Pierce, K.; Schumann, C. M.; Redcay, E.; Buckwalter, J. A.; Kennedy, D. \& Morgan, J. Mapping early brain development in autism. Neuron, 56(2):399-413, 2007.

Courchesne, E.; Campbell, K. \& Solso, S. Brain growth across the life span in autism: age-specific changes in anatomical pathology. Brain Res., 1380:138-45, 2011.

Dager, S. R.; Wang, L.; Friedman, S. D.; Shaw, D. W.; Constantino, J. N.; Artru, A. A.; Dawson, G. \& Csernansky, J. G. Shape mapping of the hippocampus in young children with autism spectrum disorder. Am. J. Neuroradiol., 28(4):672-7, 2007.

Dalton, K. M.; Nacewicz, B. M.; Alexander, A. L. \& Davidson, R. J. Gazefixation, brain activation, and amygdala volume in unaffected siblings of individuals with autism. Biol. Psychiatry., 61(4):512-20, 2007.

de Ribaupierre, S.; Ryser, C.; Villemure, J. G. \& Clarke, S. Cerebellar lesions: is there a lateralization effect on memory deficits?. Acta Neurochirurgica, 150:545-50, 2008.

D`Mello, A. M. \& Stoodley, C. J. Cerebro-cerebellar circuits in autism spectrum disorder. Front. Neurosci., 9:408, 2015.

Dorris, L.; Espie, C. A.; Knott, F. \& Sal, J. Mind-reading difficulties in the siblings of people with Asperger's syndrome: evidence for a genetic influence in the abnormal development of a specific cognitive domain. J. Child. Psychol. Psychiatry., 45(2):412-8, 2004.

Duerden, E. G.; Mak-Fan, K. M.; Taylor, M. J. \& Roberts, S. W. Regional differences in grey and white matter in children and adults with autism spectrum disorders: an activation likelihood estimate (ALE) metaanalysis. Autism Res., 5(1):49-66, 2012.

Exner, C.; Weniger, G. \& Irle, E. Cerebellar lesions in the PICA but not the SCA territory impair cognition. Neurology, 63(11):2132-5, 2004.

Fatemi, S. H.; Aldinger, K. A.; Ashwood, P.; Bauman, M. L.; Blaha, C. D.; Blatt, G. J.; Chauhan, A.; Chauhan, V.; Dager, S. R.; Dickson, P. E.; Estes, A. M.; Goldowitz, D.; de Heck, D. H.; Kemper, T. L.; Rey, B. H.; Martin, L. A.; Millen, K. J.; Mittleman, G.; Mosconi, M. W.; Persico, A. M.; Sweeney, J. A.; Webb, S. J. \& Welsh, J. P. Consensus paper: pathological role of the cerebellum in autism. Cerebellum, 11(3):777807, 2012.

Fidler, D. J.; Bailey, J. N. \& Smalley, S. L. Macrocephaly in autism and other pervasive developmental disorders. Dev. Med. Child. Neurol.,
42(11):737-40, 2000

Fombonne, E.; Rogé, B.; Claverie, J.; Courty, S. \& Frémolle, J. Microcephaly and macrocephaly in autism. J. Autism Dev. Disord., 29(2):113-9, 1999.

Ferrucci, R.; Marceglia, M.; Vergari, M.; Cogiamanian, F.; Mrakic-Sposta, S.; Mameli, F.; Zago, S.; Barbieri, S. \& Priori A. Cerebellar transcranial direct current stimulation impairs the practice-dependent proficiency increase in working memory. J. Cogn. Neurosci., 20(9):1687-97, 2008.

Ghaziuddin, M. Should DSM V drop Asperger syndrome?. J. Autism Dev. Disord., 40(9):1146-8, 2010.

Glickstein, M.; Strata, P. \& Voogd, J. Cerebellum: history. Neuroscience, 162(3):549-59, 2009.

Gottwald, B.; Wilde, B.; Mihajlovic, Z. \& Mehdorn, H. M. Evidence for distinct cognitive déficits after focal cerebellar lesions. J. Neurol. Neurosurg. Psychiatry, 75(11):1524-31, 2004.

Gowen, E. \& Miall, C. R. Behavioural aspects of cerebellar function in adults with Asperger syndrome. Cerebellum, 4(4):279-89, 2005.

Giedd, J. N.; Vaituzis, A. C.; Hamburger, S. D.; Lange, N.; Rajapakse, J. C.; Kaysen, D.; Vauss, Y. C. \& Rapoport, J. L. Quantitative MRI of the temporal lobe, amygdala, and hippocampus in normal human development: ages 4-18 years. J. Comp. Neurol., 366(2):223-30, 1996.

Groen, W.; Teluij, M.; Buitelaar, J. \& Tendolkar, I. Amygdala and hippocampus enlargement during adolescence in autism. J. Am. Acad. Child. Adolesc. Psychiatry, 49(6):552-60, 2010.

Happé, F. Why fold Asperger syndrome into Autism Spectrum Disorder in the DSM-5? (Simons Foundation Autism Research Initiative), 2011. Obtenido de: https://spectrumnews.org/opinion/viewpoint/why-fold-asperger-syndrome-into-autism-spectrum-disorder-in-the-dsm-5

Hardan, A. Y.; Minshew, N. J.; Harenski, K. \& Keshavan, M. S. Posterior fossa magnetic resonance imaging in autism. J. Am. Acad. Child. Adolesc. Psychiatry, 40(6):666-672, 2001.

Haxby, J. V.; Hoffman, E. A. \& Gobbini, M.I. Human neural systems for face recognition and social communication. Biol. Psychiatry, 51(1):5967, 2002.

Haznedar, M. M.; Buchsbaum, M. S.; Hazlett, E. A.; LiCalzi, E. M.; Cartwright, C. \& Hollander, E. Volumetric analysis and three-dimensional glucose metabolic mapping of the striatum and thalamus in patients with autism spectrum disorders. Am. J. Psychiatry, 163(7):125263, 2006.

Hazlett, H.C.; Poe, M.; Gerig, G.; Smith, R.G.; Provenzale, J.; Ross, A.; Gilmore, J. \& Piven, J. Magnetic resonance imaging and head circumference study of brain size in autism: birth through age 2 years. Arch. Gen. Psychiatry, 62(12):1366-76, 2005.

Hyde, K. L.; Samson, F.; Evans, A. C. \& Mottron, L. Neuroanatomical differences in brain areas implicated in perceptual and other core features of autism revealed by cortical thickness analysis and voxel-based morphometry. Hum. Brain Mapp, 31(4):556-66, 2010.

Hokkanen, L. S. K.; Kauranen, V.; Roine, R. O.; Salonen, O. \& Kotila, M. Subtle cognitive deficits after cerebellar infarcts. Eur. J. Neurol., 13(2):161-70, 2006

Holt, R. J.; Chura, L. R.; Lai, M. C.; Suckling, J.; von dem Hagen, E.; Calder, A. J.; Bullmore, E. T.; Baron-Cohen, S. \& Spencer, M. D. 'Reading the mind in the eyes': an fMRI study of adolescents with autism and their siblings. Psychol. Med., 44(15):3215-27, 2014.

Howlin, P. Outcome in high-functioning adults with autism with and without early language delays: implications for the differentiation between autism and Asperger syndrome. J. Autism. Dev. Disord., 33(1):3-13, 2003.

Jin, J. \& Maren, S. Prefrontal-hippocampal interactions in memory and emotion. Fronte Syst. Neurosci., 9:170, 2015.

Jumah, F.; Ghannam, M.; Jaber, M.; Adeeb, N. \& Tubbs, R. Neuroanatomical variation in autism spectrum disorder: a comprehensive review. Clin. Anat., 29(4):454-65, 2016.

Kalmbach, B. E.; Ohyama, T.; Kreider, J. C.; Riusech, F. \& Mauk, M. D. Interactions between prefrontal cortex and cerebellum revealed by trace eyelid conditioning. Learn Mem., 16(1):86-95, 2009. 
Kaufmann, W. E.; Cooper, K. L.; Mostofsky, S. H.; Capone, G. T.; Kates, W. R.; Newschaffer, C. J.; Bukelis, I.; Stump, M. H.; Jann, A. E. \& Lanham, D. C. Specificity of cerebellar vermian abnormalities in autism: a quantitative magnetic resonance imaging study. J. Child Neurol., 18(7):463-70, 2003.

Kanner L. Autistic disturbances of affective contact. Acta Paedopsychiatr. 35(4):100-36, 1968

Kemper, T. L. \& Bauman, M. Neuropathology of infantile autism. J. Neuropathol. Exp. Neurol.,57(7):645-52, 1998.

Klin, A.; Pauls, D.; Schultz, R. \& Volkmar, F. Three diagnostic approaches to Asperger syndrome: implications for research. J. Autism. Dev. Disord., 35:221-34, 2005.

Kelly, R. M. \& Strick, P. L. Cerebellar loops with motor cortex and prefrontal cortex of a nonhuman primate. J. Neurosci., 23 (23):8432-44, 2003.

Koolschijn, P. C. \& Geurts, H. M. Gray matter characteristics in mid and old aged adults with ASD. J. Autism Dev. Disord., 46(8):2666-78, 2016.

Kwon, H.; Ow, A. W.; Pedatella, K. E.; Lotspeich, L. J. \& Reiss, A. L. Voxelbased morphometry elucidates structural neuroanatomy of highfunctioning autism and Asperger syndrome. Dev. Med. Child. Neurol., 46(11):760-4, 2004

Lai, M. C.; Lombardo, M. V.; Ecker, C.; Chakrabarti, B.; Suckling, J.; Bullmore, E. T.; Happé, F.; MRC AIMS Consortium; Murphy, D. G. \& Baron-Cohen, S. Neuroanatomy of individual differences in language in adult males with autism. Cereb. Cortex, 25(10):3613-28, 2015.

Leggio, M. G.; Tedesco, A. M.; Chiricozzi, F. R.; Clausi, S.; Orsini, A. \& Molinari, M. Cognitive sequencing impairment in patients with focal or atrophic cerebellar damage. Brain, 131(Pt 5):1332-43, 2008.

Li, D.; Karnath, H \& Xu, X. Candidate biomarkers in children with autism spectrum disorder: a review of MRI studies. Neurosci. Bull., 33(2):21937, 2017.

Lichtenstein, P.; Carlström, E.; Råstam, M.; Gillberg, C. \& Anckarsäter, H. The genetics of autism spectrum disorders and related neuropsychiatric disorders in childhood. Am. J. Psychiatry 167(11):1357-63, 2010.

Lidzba, K.; Wilke, M.; Staudt, M.; Krageloh-Mann, I. \& Grood, W. Reorganization of the cerebrocerebellar network of language production in patients with congenital left-hemisphere brain lesions. Brain Lang., 106(3):204-10, 2008.

Liu, R. S.; Lemieux, L.; Bell, G. S.; Sisodiya, S. M.; Shorvon, S. D.; Sander, J. W. \& Duncan, J. S. A longitudinal study of brain morphometrics using quantitative magnetic resonance imaging and difference image analysis. Neuroimage, 20(1):22-33, 2003.

Lotspeich, L. J.; Kwon, H.; Schumann, C. M.; Fryer, S. L.; Goodlin-Jones, B. L.; Buonocore, M. H.; Lammers, C. R.; Amaral, D. G. \& Reiss, A L. Investigation of neuroanatomical differences between autism and Asperger syndrome. Arch. Gen. Psychiatry, 61(3):291-8, 2004.

Macintosh, K. \& Dissanayake, C. A comparative study of the spontaneous social interactions of children with high-functioning autism and children with Asperger's disorder. Autism, 10(2):199-220, 2006.

McAlonan, G. M.; Daly, E.; Kumari, V.; Critchley, H. D.; van Amelsvoort, T.; Suckling, J.; Simmons, A.; Sigmundsson, T.; Greenwood, K.; Russell, A.; Schmitz, N.; Happe, F.; Howlin, P. \& Murphy, D. G. Brain anatomy and sensorimotor gating in Asperger's syndrome. Brain, 125 (Pt 7):1594606, 2002.

McAlonan, G. M.; Cheung, V.; Cheung, C.; Suckling, J.; Lam, G. Y.; Tai, K. S.; Yip, L.; Murphy, D. G. \& Chua, S. E. Mapping the brain in autism. A voxel-based MRI study of volumetric differences and intercorrelations in autism. Brain, 128(Pt 2):268-76, 2005.

McAlonan, G. M.; Suckling, J.; Wong, N.; Cheung, V.; Lienenkaemper, N.; Cheung, C.; Chua, S. E. Distinct patterns of grey matter abnormality in high- functioning autism and Asperger's syndrome. J. Child Psychol. Psychiatry, 49(12):1287-95, 2008.

McAlonan, G.M.; Cheung, C.; Cheung, V.; Wong, N.; Suckling, J. \& Chua, S. E. Differential effects on white-matter systems in high-functioning autism and Asperger's syndrome. Psychol. Med., 39(11):1885-93, 2009.

Mattfeld, A. T. \& Stark, C. E. Functional contributions and interactions between the human hippocampus and subregions of the striatum during arbitrary associative learning and memory. Hippocampus, 25(8):900-11, 2015.

Mayes, S. D.; Calhoun, S. L. \& Crites, D. L. Does DSM-IV Asperger's disorder exist?. J. Abnorm. Child. Psychol., 29(3):263-71, 2001.

Middleton, F. A. \& Strick, P. L. Basal ganglia and cerebellar loops: motor and cognitive circuits. Brain Res. Brain Res. Rev., 31(2-3):236-50, 2000.

Middleton, F. A. \& Strick, P. L. Cerebellar projections to the prefrontal cortex of the primate. J. Neurosci., 21(2):700-12, 2001.

Montgomery, C. B.; Allison, C.; Lai, M. C.; Cassidy, S.; Langdon, P. E. \& Baron-Cohen, S. Do adults with high functioning autism or Asperger syndrome differ in empathy and emotion recognition?. J. Autism Dev. Disord., 46(6):1931-40, 2016.

Mosconi, M. W..; Cody-Hazlett, H.; Poe, M. D.; Gerig, G.; Gimpel-Smith, R. \& Piven J. Longitudinal study of amygdala volume and joint attention in 2- to 4-year-old children with autism. Arch. Gen. Psychiatry, 66(5):50916, 2009.

Murphy, C. M.; Deeley, Q.; Daly, E. M.; Ecker, C.; O’Brien, F. M.; Hallahan, B.; Loth, E.; Toal, F.; Reed, S.; Hales, S.; Robertson, D. M.; Craig, M. C.; Mullins, D.; Barker, G. J.; Lavender, T.; Johnston, P.; Murphy, K. C. \& Murphy, D. G. Anatomy and aging of the amygdala and hippocampus in autism spectrum disorder: an in vivo magnetic resonance imaging study of Asperger syndrome. Autism Res., 5:3-12, 2012.

Nickl-Jockschat, T.; Habel, U.; Michel, T. M.; Manning, J.; Laird, A. R.; Fox, P. T.; Schneider, F. \& Eickhoff, S. B. Brain structure anomalies in autism spectrum disorder--a meta-analysis of VBM studies using anatomic likelihood estimation. Hum. Brain Mapp., 33(6):1470-89, 2012.

O'Brien, F. M.; Página, L.; O'Gorman, R. L.; Bolton.; P.; Sharma, A.; Baird, G.; Daly, E.; Hallahan, B.; Conroy, R. M.; Foy, C.; Curran, S.; Robertson, D.; Murphy, K. C. \& Murphy, D. G. Maturation of limbic regions in Asperger syndrome: a preliminary study using proton magnetic resonance spectroscopy and structural magnetic resonance imaging. Psiquiatria Res., 184(2):77-85, 2010.

Ozonoff, S.; South, M. \& Miller, J. N. DSM-IV-defined Asperger syndrome: cognitive, behavioral and early history differentiation from highfunctioning autism. Autism, 4(1):29-46, 2000.

Palmen, S. J.; Hulshoff Pol, H. E.; Kemner, C.; Schnack, H. G.; Durston, S.; Lahuis, B. E.; Kahn, R. S.; \& Van Engeland, H. Increased graymatter volume in medication-naïve high-functioning children with autism spectrum disorder. Psychol. Med., 35(4):561-70, 2005.

Pierce, K. \& Courchesne, E. Evidence for a cerebellar role in reduced exploration and stereotyped behavior in autism. Biol. Psychiatry, 49(8):655-64, 2001.

Pierce, K.; Müller, R. A.; Ambrose, J.; Allen, G. \& Courchesne, E. Face processing occurs outside the fusiform 'face area' in autism: evidence from functional MRI. Brain, 124(Pt 10):2059-73, 2001.

Pierce, K.; Haist, F.; Sedaghat, F. \& Courchesne, E. The brain response to personally familiar faces in autism: findings of fusiform activity and beyond. Brain, 127(Pt 12):2703-16, 2004.

Pina-Camacho, L.; Villero, S.; Boada, L.; Fraguas, D.; Janssen, J.; Mayoral, M.; Llorente, C.; Arango, C. \& Parellada, M. Structural magnetic resonance imaging data do not help support DSM-5 autism spectrum disorder category. Res. Autism Spectr. Disord., 7(2):333-43, 2013.

Piven, J.; Saliba, K.; Bailey, J. \& Arndt, S. An MRI study of autism: the cerebellum revisited. Neurology, 49(2):546-51, 1997.

Planche, P. \& Lemonnier, E. Research in autism spectrum disorders Children with high-functioning autism and Asperger's syndrome: can we differentiate their cognitive profiles?. Res. autismo Spectr. Disorders, 6(2):939-48, 2012.

Pruessner, J. C.; Collins, D. L.; Pruessner, M. \& Evans, A. C. Age and gender predict volume decline in the anterior and posterior hippocampus in early adulthood. J. Neurosci., 21(1):194-200, 2000.

Raymond, G. V.; Bauman, M. L. \& Kemper, T. L. The hippocampus in autism: golgi analysis. Acta Neuropathol., 91(1):117-9, 1989.

Raz, N.; Lindenberger, U.; Rodrigue, K. M.; Kennedy, K. M.; Head, D.; Williamson, A.; Dahle, C.; Gerstorf, D. \& Acker, J. D. Regional brain changes in aging healthy adults: general trends, individual differences 
and modifiers. Cereb. Cortex, 15(11):1676-89, 2005

Richardson, M. P.; Strange, B. A. \& Dolan, R. J. Encoding of emotional memories depends on amygdala and hippocampus and their interactions. Nat. Neurosci., 7(3):278-85, 2004.

Redcay, E. \& Courchesne, E. When is the brain enlarged in autism? A meta-analysis of all brain size reports. Biol. Psychiatry, 58(1):1-9, 2005.

Richell, R. A.; Mitchell, D. G. V.; Newman, C.; Leonard, A.; Baron- Cohen, S. \& Blair, R. J. R. Theory of mind and psychopathy: Can psychopathic individuals read the "language of the eyes"? Neuropsychologia, 41(5):523-6, 2003.

Richter-Levin, G. The amygdala, the hippocampus, and emotional modulation of memory. Neuroscientist., 10(1):31-9, 2004.

Rogers, T. D.; McKimm, E.; Dickson, P. E.; Goldowitz, D.; Blaha, C. D. \& Mittleman G. Is autism a disease of the cerebellum? An integration of clinical and pre-clinical research. Front. Syst. Neurosci., 7:15, 2013.

Ruggeri, B.; Sarkans, U.; Schumann, G. \& Persico, A. M. Biomarkers in autism spectrum disorder: the old and the new. Psychopharmacology (Berl), 231(6):1201-16, 2014.

Saitoh, O.; Karns, C. M. \& Courchesne, E. Development of the hippocampal formation from 2 to 42 years: MRI evidence of smaller area dentata in autism. Brain, 124(Pt 7):1317-24, 2001.

Salmond, C. H.; Ashburner, J.; Connelly, A.; Friston, K. J.; Gadian, D. G. \& Vargha-Khadem, F. The role of the medial temporal lobe in autistic spectrum disorders. Eur. J. Neurosci., 22(3):764-72, 2005.

Schmahmann, J. D. The cerebrocerebellar system: anatomic substrates of the cerebellar contribution to cognition and emotion. Int. Rev. Psychiatry, 13(4):247-60, 2001

Schumann, C. M.; Hamstra, J.; Goodlin-Jones, B. L.; Lotspeich, L. J.; Kwon, H.; Buonocore, M. H.; Lammers, C. R.; Reiss, A. L. \& Amaral, D.G. The amygdala is enlarged in children but not adolescents with autism; the hippocampus is enlarged at all ages. J. Neurosci., 24(28):6392-401, 2004.

Schumann, C. M.; Barnes, C. C.; Lord, C. \& Courchesne, E. Amygdala enlargement in toddlers with autism related to severity of social and communication impairments. Biol. Psychiatry, 66(10):942-9, 2009.

Schumann, C. M.; Bloss, C. S.; Barnes, C. C.; Wideman, G. M.; Carper, R. A.; Akshoomoff, N.; Pierce, K.; Hagler, D.; Schork, N.; Lord, C. \& Courchesne, E. Longitudinal magnetic resonance imaging study of cortical development through early childhood in autism. J. Neurosci., 30:4419-27, 2010.

Schweizer, T. A.; Alexander, M. P.; Cusimano, M. \& Stuss, D. T. Fast and efficient visuotemporal attention requires the cerebellum. Neuropsychologia, 45(13):3068-74, 2007.

Sigman, M.; Mundy, P.; Sherman, T. \& Ungerer, J. Social interactions of autistic, mentally retarded and normal children and their caregivers. $J$. Child. Psychol. Psychiatry, 27:647-55, 1986.

Sparks, B. F.; Friedman, S. D.; Shaw, D. W.; Aylward, E. H.; Echelard, D.; Artru, A. A.; Maravilla, K. R.; Giedd, J. N.; Munson, J.; Dawson, G. \& Dager, S. R. Brain structural abnormalities in young children with autism spectrum disorder. Neurology, 59(2):184-92, 2002.

Spek, A. A.; Scholte, E. M.; \& Berckelaer-Onnes, I. A. Theory of Mind in adults with HFA and Asperger syndrome. J. Autism Dev. Disord., 40(3):280-9, 2009.

Strick, P. L.; Dum, R. P. \& Fiez, J. A . Cerebellum and nonmotor function. Annu. Rev. Neurosci., 32:413-34, 2009.

Suzuki, M.; Hagino, H.; Nohara, S.; Zhou, S. Y.; Kawasaki, Y.; Takahashi, T; Matsui, M.; Seto, H.; Ono, T. \& Kurachi M. Male-specific volume expansion of the human hippocampus during adolescence. Cereb. Cortex, 15(2):187-93, 2005.

Toal, F.; Daly, E. M.; Page, L.; Deeley, Q.; Hallahan, B.; Bloemen, O.; Cutter, W. J.; Brammer, M. J.; Curran, S.; Robertson, D.; Murphy, C.; Murphy, K. C. \& Murphy, D. G. Clinical and anatomical heterogeneity in autistic spectrum disorder: a structural MRI study. Psychol. Med., 40(7):1171-81, 2010.

Townsend, J.; Courchesne, E.; Covington, J.; Westerfield, M.; Harris, N. S.; Lyden, P.; Lowry, T. P. \& Press, G. A. Spatial attention deficits in patients with ac- quired or developmental cerebellar abnormality. $J$. Neurosci., 19(13):5632-43, 1999.

Tulving, E. Memory and consciousness. Can. Psychol., 26(1):1-12, 1985. Vargas, D. L.; Nascimbene, C.; Krishnan, C.; Zimmerman, A. W. \& Pardo, C. A. Neuroglial activation and neuroinflammation in the brain of patients with autism. Ann. Neurol. 57(1):67-81, 2005.

Volkmar, F. R. \& Partland, J. C. From Kanner to DSM-5: Autism as an evolving diagnostic concept. Annu. Rev. Clin. Psychol., 10:193-212, 2014.

Wallace, G. L.; Dankner, N.; Kenworthy, L.; Giedd, J. N. \& Martin, A. Age-related temporal and parietal cortical thinning in autism spectrum disorders. Brain, 133(Pt 12):3745-54, 2010.

Webb, S. J.; Sparks, B. F.; Friedman, S. D.; Shaw, D. W.; Giedd, J.; Dawson, G. \& Dager, S. R. Cerebellar vermal volumes and behavioral correlates in children with autism spectrum disorder. Psychiatry Res., 172(1):617, 2009.

Welchew, D. E.; Ashwin, C.; Berkouk, K.; Salvador, R.; Suckling, J.; BaronCohen, S. \& Bullmore, E. Functional disconnectivity of the medial temporal lobe in Asperger's Syndrome. Biol. Psychiatry, 57(9):991-8, 2005.

Whitney, E. R.; Kemper, T. L.; Bauman, M. L.; Rosene, D. L. \& Blatt, G. J. Cerebellar Purkinje cells are reduced in a subpopulation of autistic brains: a stereological experiment using calbindin-D28k. Cerebellum, 7(3):406-16, 2008.

Williams, R. S.; Hauser, S. L.; Purpura, D. P.; DeLong, G. R. \& Swisher, C. N. Autism and mental retardation: neuropathologic studies performed in four retarded persons with autistic behavior. Arch. Neurol., 37(12):749-53, 1980.

Wing, L. Asperger's syndrome: a clinical account. Psychol. Med., 11(1):11529, 1981.

Wing, L. The relation between Asperger's syndrome and Kanner's autism. Autism and Asperger Syndrome, 93-121, 1994.

Witwer, A. N. \& Lecavalier, L. Examining the validity of autism spectrum disorder subtypes. J. Autism Dev. Disord., 38(9):1611-24, 2008.

World Health Organization (WHO). The ICD-10 classification of mental and behavioural disorders. Clinical descriptions and guidelines. Geneva: WHO, 1994.

Yu, K. K.; Cheung, C.; Chua, S. E.; \& McAlonan, G. M. Can Asperger syndrome be distinguished from autism? An anatomic likelihood metaanalysis of MRI studies. J. Psychiatry Neurosci., 36(6):412-21, 2011.

\section{Dirección para Correspondencia: \\ Dra. Bélgica Vásquez P. \\ Facultad en Ciencias de la Salud \\ Universidad de Tarapacá \\ Arica \\ CHILE}

E-mail: bvasquezp@uta.cl

Recibido : 09-09-2016

Aceptado: 11-12-2016 